\title{
A Configurational Approach for Assessing Organizational Effectiveness of the Architectural/Construction Firm
}

\author{
Adnan Abbas Adas \\ Department of Architecture, Faculty of Environmental Design, \\ King Abdulaziz University, Jeddah, Saudi Arabia
}

\begin{abstract}
AвstRact. An assessment method of organizational effectiveness which considers the important organizational attributes of the architectural/construction firm is described. The method is based on a validated configurational approach identified as the competing values approach. In this paper, theoretical approaches used to model organizational effectiveness and the logic of using the selected approach is discussed. The selected approach consolidates most criteria of effectiveness into four ideal models each with dominant criteria that are pursued by the firm during its various life cycle stages. These criteria are utilized to define four groups of organizational variables relevant to examining effectiveness: structural context; personoriented processes; strategic means; and organizational flexibility, rules and regulations. Different levels of these variables occurring together are hypothesized to define multiple configurations that are pursued by firms in their quest for effectiveness. Once the configuration pursued by the firm is identified, the existing levels of variables in the firm can be compared to those in the configuration to yield a reliable assessment. Further research steps are outlined as how to validate such a methodology.
\end{abstract}

Keywords: Configurational approach, effectiveness, assessment, architectural/construction firm.

\section{Introduction}

Management in architectural/construction firms must address two important issues related to organizational effectiveness and performance: what theoretical approach or model is most appropriate to define and understand it? And based on that, how to develop a suitable methodology to assess effectiveness relative to their scope of operation. 
The study of organizational effectiveness represents a complex part of organization theory due to the various approaches that can be utilized to model it. Researchers have offered a variety of models for examining effectiveness, yet there is little consensus as to what constitutes a valid set of effectiveness criteria ${ }^{[1]}$. In the literature many criteria and techniques were used in developing measurement. For example traditional techniques apply common approaches such as the goal model used by Pennings \& Goodman in their measurement framework ${ }^{[2]}$. Other techniques such as Hitt's rely on the more elaborate systems approaches using models such as the resource model or strategic adaptation mode ${ }^{[3]}$.

In general, most commonly used methodologies are based on the goal approach. They measure the level of achievement of a specific goal or goals. Three commonly used indicators in architectural/construction projects ask if work was completed on time, within budget, and if it met certain quality standards. Other indicators measure levels of achieved profits and/or levels of workers' productivity and compare these levels to specific levels that were established as goals. Profit indicators by themselves are crude and shortsighted. Clearly a firm can make a profit without being effective by cutting corners and improper practices; such firms do not stay in business long. These indicators do not give enough information about organizational effectiveness of the firm. Improper assessment of effectiveness by firms lead to inaccurate conclusions which in turn result in sub-standards performance. Although most managers use some indicators (mostly financial), these do not capture all of the salient elements of effectiveness and can not be relied upon as predictors of effectiveness. Development of better methods to measure effectiveness should be a high priority in order to achieve and maintain improved performance.

\section{Effectiveness}

There are various views of effectiveness advanced by different theorists that compete for the attention of researchers, each has its strength and weakness. Theorists of the classical school, developed universal principles or models that would apply in all situations and treated firms as closed systems. For example, Weber's theory of bureaucracy that identified characteristics of bureaucratic authority in the organization, how formalized rules and regulation are used to govern and control the firm through hierarchically structured positions in order to achieve effectiveness. One of the principal drawbacks of classical theorists is that they tend to treat all firms as machine like closed systems and that organizational control and hence effectiveness can be achieved by division of work, establishing lines of authority, and discipline. Influence of external environment is not recognized. 
The classical view was later challenged by some theorists who advanced the social nature of organizations. They proposed that management's roles in achieving effectiveness were to facilitate communication and to stimulate subordinates to high level of effort. An example here is the human-relation school with its participative decision-making principles which emerged from McGregor's Theory X- Theory Y which promotes the creation of responsible jobs for workers and developing good group or team relations.

Afterwards, an open-system perspective for examining the functioning of the firm and its effectiveness emerged. Systems approaches emphasize the organization as a system and attempt to assess the effectiveness of the system in terms of its inputs, transformation, and outputs. Examples here include the internal process model and the strategic adaptation model. In the internal process model, effectiveness is reflected in the efficiency of the processes inside the organizational systems. Theoretically, looking at the internal processes of the system, especially when the firm has little control over its environments seems to be more revealing of the effectiveness of the system than any other approach. However the approach has a narrow perspective of the functioning of the organization. It has no focus on the external interactions of the firm. Strategic adaptation model suggests that effective firms monitor their external environment constantly, receive feed back regularly, and take corrective actions to achieve their goals in the short term and ensure survival in the long term. This model recognizes the open-system nature of organizations, and their susceptibility to external forces. A major limitation of this model is that it pays little attention to what goes on inside the organization.

Another perspective advanced that in order for firms to achieve organizational effectiveness, they should study their conditions or the environment in which they function. This perspective is known as contingency theory. It states that effective design of organizational structures' parameters (such as job specialization, unit size, centralization, etc.) are contingent or influenced by the various characteristics of its environment such as complexity and stability; the age and size of the organization; its technical system (technology) used for production; and its power system, for example, which controls the organization. Many contingency theorists have investigated environment-structure relationship and defined many types of environments and organizational structures that best fit for them.

Yet, another perspective draws attention to the various stockholders or constituencies in the internal and external environment around the organization. Stakeholders are groups or individuals affected by the organization performance who seek to influence the organization to satisfy their goals. Stakeholders could include any number of the groups that include owners of the organization, government and regulating agencies, local community organizations, customers, 
competitors, workers, special interest groups, environmentalists, suppliers, and the media. This perspective is based on the political view of the organization and it integrates the criterion of effectiveness for each group or constituency within or outside the organization that has a stake in the firm's performance. An example here is the strategic constituencies model. A major limitation of this model is assignment of proper weights to constituents to indicate the relative importance of satisfying their goals ${ }^{[4]}$.

According to contingency theory firms must change their internal attributesstructures, strategies, and processes-to cope with changes in the environment. It is argued that organizations opt for internal consistency or stable configurations as long as possible because of reasons that include: (a) environmental change can sometimes prove to be temporary and therefore it is sensible to delay reaction to it; (b) internal changes are costly and therefore it will be resisted especially when a successful integration of structural and process attributes have been achieved; and finally successful organizations are never sure of the attributes that lie at the roots of their success and thus would avoid tampering with their tried and successful configuration ${ }^{[5]}$. Usually adaptation is avoided until a major threat is perceived because change must eventually come. In the face of worsening environmental fit, firms opt for totally new organizational configurations changing all their attributes drastically rather than piecemeal attributes change.

In the literature, a number of configurations were uncovered such as "mechanistic" structures in firms dealing with stable environments and "organic" structures in firms found in dynamic environments. The prospector, the analyzer, the defender, and the reactor forms of organization resulted from classifying the organizations according to their strategies, structures, and managerial styles ${ }^{[6]}$. The effective structuring of organizations into five configurations by Mintzberg that include: the simple structure where force of direction that the various activities of an organization take to achieve a common goal, and results in the entrepreneurial form when this force dominates an organization; the machine bureaucracy where the force for efficiency becomes dominant, which attempts to ensure a viable ratio of benefits gained to costs incurred; the professional bureaucracy where the force of proficiency is dominant, which makes organization carry out tasks with high knowledge and skill; the divisionalized form where the force for concentration helps concentrate efforts on serving particular markets; and the adhocracy form that develops in response to an overriding need to innovate a new product ${ }^{[7]}$.

Organizational effectiveness study according to configurations is justified on grounds of attempting to understand commonalities across organizations that make them effective. Organizations are driven toward configurations in order to achieve consistency in their characteristics and rather than trying to do well on 
everything, effective organizations concentrate on configurations and try to bring their elements into line with these configuration ${ }^{[8]}$. A configurational approach that models organizational effectiveness criteria pursued by business firms has been proposed and is known as the competing values ${ }^{[9]}$. It identifies ideal configurations or types based on dominant values of structural context, focus, and strategic means and ends. An organization can pursue the values of these ideal configurations and depending how close it is to these values determines its effectiveness.

\section{The Competing Values Approach}

The competing values approach is based on the premise that there is no one criterion for evaluating effectiveness. It organizes, consolidates, and integrates multiple criteria in the domains of effectiveness into three sets of incompatible dimensions. These are flexibility versus control, internal versus external focus, and means versus ends. The first set contrasts two dimensions of an organization's structure: flexibility values innovations, adaptation, and change while control favors stability, order, and predictability. The second set deals with whether focus and emphasis should be placed internally on the well-being and development of the people in the organization or externally on the well-being of the organization itself. The third set relates to organizational means versus ends; the former stressing internal processes and the long term, the latter emphasizing final outcomes and the short term.

These three sets are depicted into four organizational models or configurations shown in Fig. 1. The models are the open system model, the humanrelations model, the rational goal model, and the internal process model. In the figure there are axes of contrasting values that define the four models. Each model represents a particular set of values and has a polar opposite with contrasting emphasis. The vertical axis pertains to organization structural context and it contrasts stability and control with flexibility. The horizontal axis pertains to focus, which is whether dominant values are internal or external to the organization. The two inner axes pertain to organizational means and ends for each model and they contrast the processes or means (e.g. goal setting) to organizational outcomes and the outcomes or ends (e.g. productivity) themselves.

In brief, each model has characteristics that differ from the other and which influence the level of effectiveness in the firm differently. The rational goal model emphasizes control and organizational focus as dominant effectiveness values; planning and goal setting are means, and productivity and efficiency are ends. The open system model emphasizes flexibility and an organizational focus as dominant effectiveness values; readiness and flexibility are means, growth and external support are ends. Dominant effectiveness values for the in- 


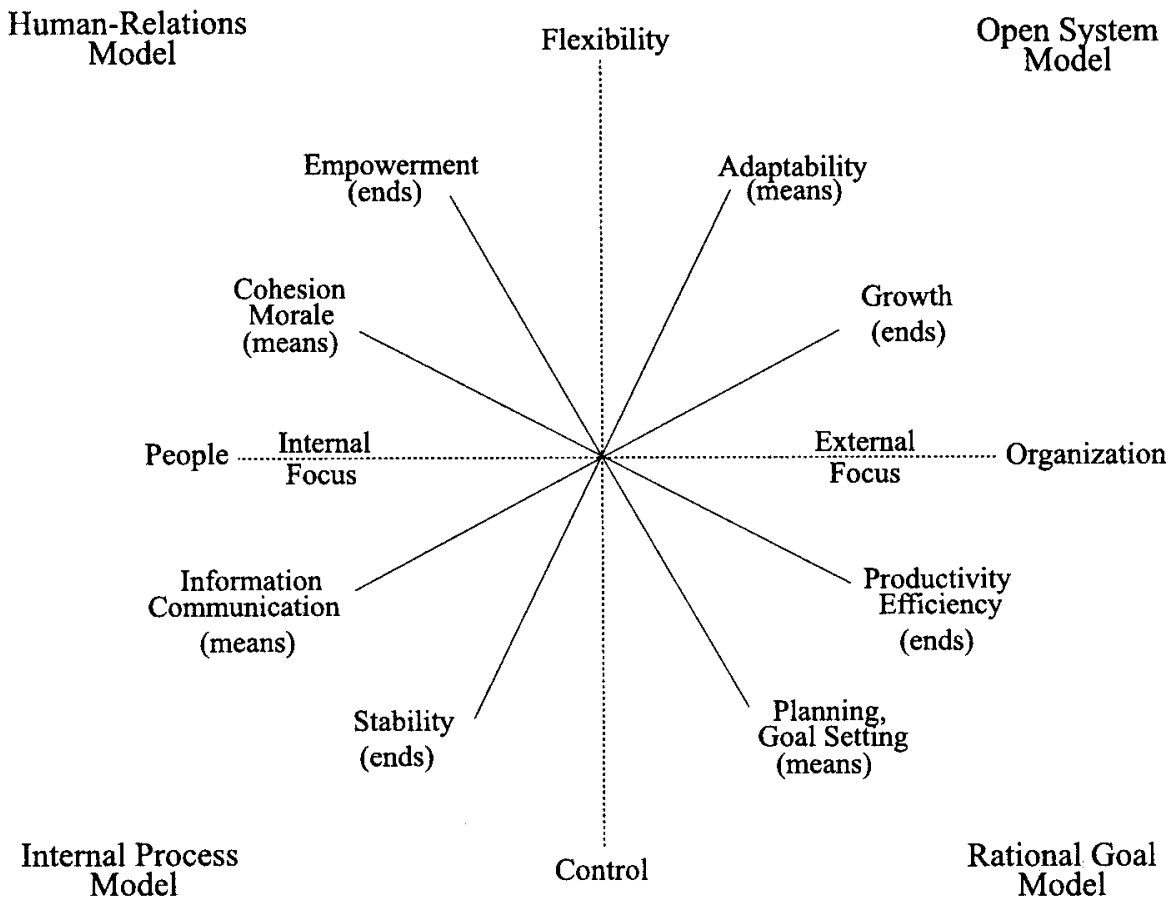

FIG. 1. Four models in the competing values approach and their effectiveness criteria.

(Source: Maloney and Federle, Practical Models For Organizational Assessment ${ }^{[11]}$.

ternal process model are control and internal focus, stressing communication processes as means and control as ends. The human-relations model emphasizes flexibility and internal focus, with cohesion and morale as means and skilled workers as ends. Firms could pursue any criteria of any one of the four models or configurations or their hybrids in their quest for effectiveness. The use of conflicting multiple criteria or characteristics is represented by the hybridization of values between the four models of the competing values approach. This hybridization results in certain tradeoffs between the different levels of conflicting or paradoxical values. For example, stressing a moderate level of competitiveness and external focus by a firm does not exclude it from placing some emphasis on the development of its workers, and adopting strategies to enhance morale and cohesion among them. This supported the inclusion of paradoxical or conflicting criteria in assessments of organizational effectiveness in order to achieve a better assessment ${ }^{[10]}$. It is argued that although firms could operate in multiple domains of effectiveness, they may also perform well only in a limited number of them. In other words, firms cannot satisfy all possible criteria of effectiveness. It is also argued that, using a multidimensional view of effectiveness implies that different patterns of relationships between organizational ef- 
fectiveness and its determinants will emerge, depending on the life cycle stage of the firm ${ }^{[4]}$.

Maloney and Federle used the competing values approach to develop a method for assessing the effectiveness of construction and engineering organizations based on assessment of configurations of organizational culture ${ }^{[11]}$. The method is based on typologies or configurations of cultures identified by the four models of the competing values approach. Assessment was achieved by classification of the organizational culture according to perceptions of management and workers along the properties of four types of cultures identified in the competing values approach. The respondents' ratings are compared with each other to check for consistency. Particular attention is placed by the method upon the comparison of the ratings of the manager and that of his subordinates because if there is a significant difference between the perceptions of the manager and his subordinates, the potential for major problems exists.

By delineating variables from the competing values four models, Ostroff \& Schmitt studied configurations of organizational effectiveness and efficiency ${ }^{[12]}$. Their findings indicate that effective and efficient organizations are influenced not only by strength of culture, but also by other variables such as participation in decision-making, goal emphasis, attitude toward change, and level of structural contextual emphasis.

Although their study did not target construction organizations, their findings suggested the inclusion of other organizational characteristics or variables along with culture in the study of effectiveness seems to be appropriate.

\section{Proposed Methodology}

The evolution of the methodologies used to study organizational effectiveness have progressed along parallel lines with the development of the various theoretical approaches. Because no single methodology is suitable for the plethora of theoretical perspectives, the key to developing a valid assessment methodology rests with understanding how the criteria of effectiveness pursued by the firm change with the evolution of the firm through the various stages of its lifecycle.

Figure 2. (a), (b), (c), and (d), shows pictograms of effectiveness criteria pursued by the firm over its various life cycle stages (only four are defined here) as suggested by Quinn and Rohrbaugh ${ }^{[9]}$. The difference from one stage to another is the change in the levels of effectiveness criteria pursued by the firm from one stage to another, as seen in states (a), (b), (c), and (d). In state (b) the firm values flexibility but somewhat less than in (d), where more emphasis is put on flexibility. During stage (a), the firm places emphasis on flexibility just as much in (d) however, it places very little emphasis on control of its processes. In (c), 
the firm places less flexibility than in (a) and (d), but places more than in (b). This view represents a more realistic model of the nature of the firm, and how it organizes itself to achieve effectiveness. This is the view considered by this research. From this perspective, a group of firms that are within the same stage of their life cycle tend to pursue similar levels of criteria of effectiveness. Therefore, effective levels of the criteria at each stage can be used to predict the organizational effectiveness of firms operating at that particular stage. Once the pursued configuration is identified, the ideal levels of effectiveness criteria in that configuration can be compared to that in the firm to yield a reliable assessment of its level of organizational effectiveness.

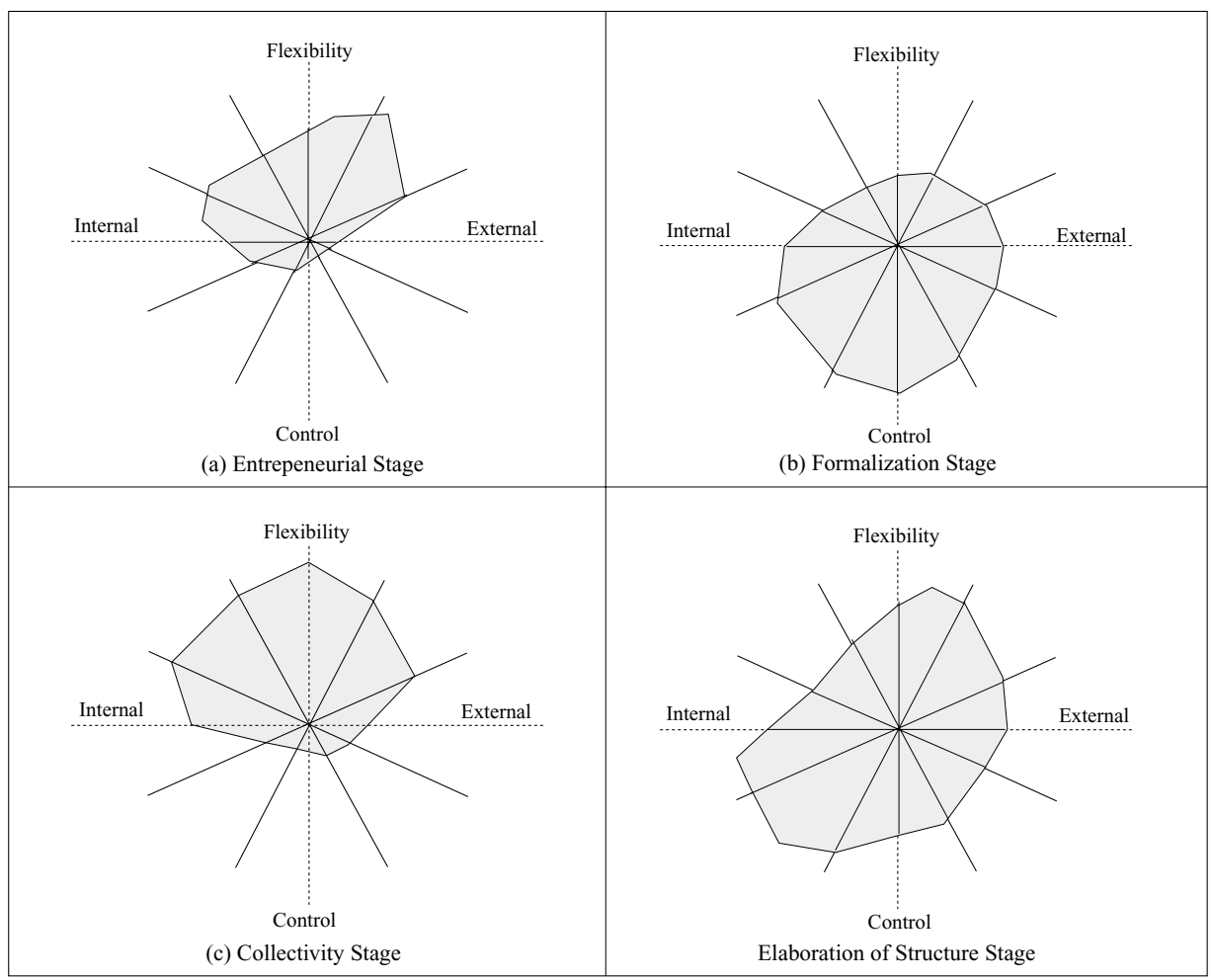

FIG. 2. Pursued levels of effectiveness criteria during firm's life cycle. (Source: A Spatial Model of Effectiveness: ${ }^{[19]}$

Three main steps are suggested in order to develop valid quantitative models based on configurational inquiry ${ }^{[13]}$. First organizational configurations in an identified approach must be conceptualized and modeled as ideal configurations where effectiveness is highest because the fit among the contextual, structural and strategic factors is at a maximum in these configurations. Second, organiza- 
tional characteristics/variables that are relevant for examining effectiveness of the firm and which represent the different effectiveness domains in the ideal configurations must be identified. Third, depending on the number of configurations defined by the utilized approach, a number of profiles with ideal levels of organizational variables must be formed. A profile must be determined empirically for each configuration to reflect the levels of the organizational variables (effectiveness criteria) in the configuration and their relationships with a valid referent measure of organizational effectiveness. Profile analysis could be used to determine the fit or deviations between the levels of criteria in the firm and their ideal levels in the pursued configuration. The degree of best fit or minimum deviation across all possible pursued configurations represent a very reliable measure of the level of organizational effectiveness of the firm (see figure 3 ).

\section{Flexibility}

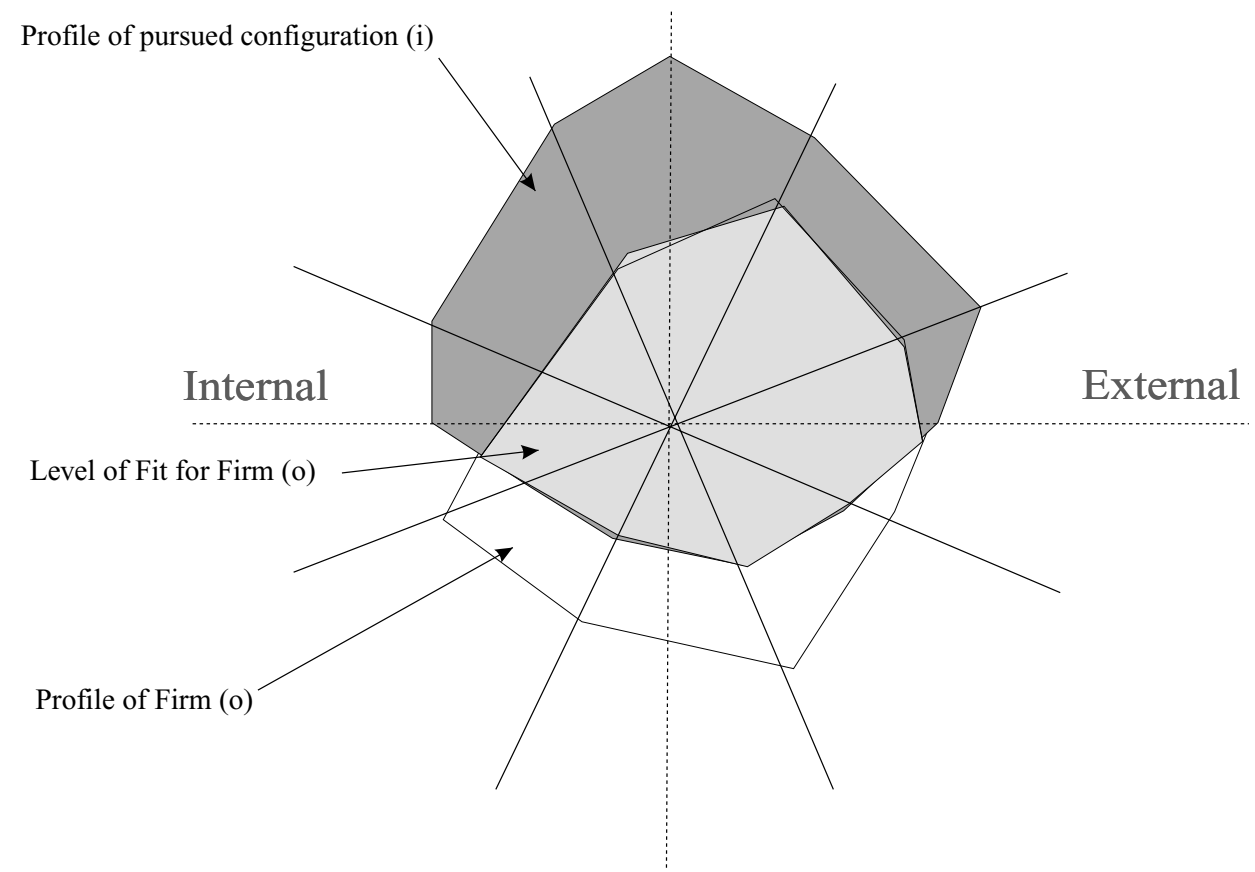

\section{Control}

FIG. 3. Fit of the levels of effectiveness criteria between the firm and the pursued configuration.

The competing values approach forms the basis of the proposed methodology. This is because the effectiveness criteria of its four models can be used to describe configurations with different levels of effectiveness criteria. A total of four configurations are hypothesized that could be pursued by the architectural/ 
construction firm. These are similar to the four configurations proposed by Quinn $^{[9]}$, and which are defined for four phases or stages of organizations life cycle (see Fig. 2). However the configurations hypothesized here for the architectural/construction firms are with different levels of effectiveness criteria. Therefore the four hypothesized configurations that may be pursued by architectural /construction firms during their life cycle in their quest for effectiveness include: a configuration that is pursued by firms during their initial or Entrepreneurial phase of lifecycle, a second configuration with different levels of effectiveness criteria pursued by firms in their Formalization phase of life cycle or growing phase, a third configuration with different levels of effectiveness criteria pursued by firms in the Collectivity phase of life cycle or maturing phase, and finally a fourth configuration pursued by firms in their Elaboration of structure phase or achieving recognition and expanding into other services or breaking-up into a number of strategic business units.

Criteria used to describe these four hypothesized configurations are identified from the four models of the competing values approach and can be grouped into the following categories: structural context; organizational flexibility, rules and regulations; organizational focus; and strategy (means vs. Ends). Analysis of architectural/construction firm's characteristics along the dimensions of effectiveness in these four categories helps to identify the important variables. In the structural context category, a number of variables can be used to indicate the effectiveness of the structural component of the architectural/construction firm in dealing with its external and internal environment. The level of subcontracting used in majority of architectural/construction projects has been shown to influence the flexibility of structure in the architectural/construction firm and reflects the firm's attitude toward risk sharing and enhancing its costs effectiveness ${ }^{[14]}$. An architectural/construction firm that pursues strategic flexibility must have a structure that is better suited for contractual arrangements that provide flexibility such as subcontracting. An architectural/construction firm, in its quest for flexibility of services offered, may integrate vertically upstream by offering financing, A/E (architectural/engineering services), and material supply services, or downstream by offering maintenance services ${ }^{[15]}$. Therefore the level of integration in services offered by the construction firm can be considered as another variable that reflects the structural flexibility of the organization in its attempt to control the quality and range of its construction product. Other variables that can be identified in this category include: level of multi-project handling which reflects the ability of the architectural/construction firm's structure to acquire resources to handle simultaneous work at different locations. 
In the second category of flexibility, rules and regulations, three variables can be used to indicate the effectiveness of the organizational structure in mediating between the flexibility-control points of the flexibility dimension. These include the extent of using regulation in work procedures, instruction, and evaluation by the firm, the level of adherence to rules and regulation by management and workers of the firm, and firm's attitude toward change. This variable would reflect management and workers' attitudes in encouraging, adopting, and accepting organizational changes in order to adapt the firm to new challenges in market.

The third category of variables is based on the dimension of focus which represents whether the firm is internally or externally focused according to the competing values approach. Internally focused architectural/construction firms that value control in the assessment of effectiveness stress adequate communication and information management through a strong culture of hierarchy with clear rules and regulations for performance. Externally focused construction firms which value flexibility as a criteria of effectiveness emphasize adaptability through a strong culture that promotes readiness and innovations. Externally focused firms which value control in the assessment of effectiveness emphasize planning, goal setting, and a strong culture that promotes productivity and accomplishment.

Two variables which are deemed important in determining the focus of the architectural/construction firm and its effectiveness are: strength of organizational culture which reflects the internal climate in the firm and its strengths or weaknesses which are directly tied to organizational effectiveness of the construction firm ${ }^{[16]}$; and level of workers' participation in decision-making which underlies firm's attempt to empower and motivate its workers. Internally focused construction firms that value flexibility in the assessment of effectiveness are more sensitive to their workers. They achieve this by allowing a higher degree of participation in decision-making through a strong culture of team work. Therefore the level of workers' participation in decision-making could be considered.

The fourth category represents strategic means used by the architectural/ construction firm to achieve effectiveness. Two important variables can be identified in this category and they are: the level of strategic planning, and the level of goal-setting. The former underscores the firm's attempt to adopt effective strategies that adapt and fit its environment. The latter reflects the level of importance that the firm puts on setting goals such as increasing profits levels, increasing cost effectiveness, growth into other construction sectors, improving level of process quality, improving client satisfaction, and increasing workers' empowerment. 
Organizational variables discussed represent the most significant variables that can be identified here along the three dimensions of the competing values approach and which can be used as criteria in the assessment of effectiveness ${ }^{[17],}[18]$. The four hypothesized configurations that are pursued by architectural/ construction firms can be deduced by studying the relationships between the levels of these variables and a valid referent measure of organizational effectiveness. A field study must be carried out to collect pertinent data from a large sample of architectural/construction firms to ensure the collection of data from a sufficient number of firms pursuing each of the four hypothesized configurations. The levels of variables in the deduced configurations can then be used as a yardstick against which their levels in the construction firm are compared. Techniques for assessing profile similarity are well developed. Most of these techniques assess deviation with some form of Euclidean distance formula:

$$
D_{i o}=\sqrt{\left(X_{i}-X_{o}\right)}
$$

where

$D_{i o}=$ the distance between levels of criteria in configuration $i$ and firm $o$

$X_{i}=\mathrm{a} 1 \mathrm{x} j$ vector that represent the level of configuration $i$ on criteria $j$

$X_{o}=\mathrm{a} 1 \mathrm{x} j$ vector represent the level of firm $o$ on criteria $j$

Under the proposed methodology a firm can adopt any one of the four hypothesized configurations depending on its stage of life cycle and remain effective. The model that should be used to assess its fit with that of the configuration it is attempting to pursue is as follows:

$$
\text { Fit } t_{o}=\left(\begin{array}{c}
I \\
\min D_{i o} \\
i=1
\end{array}\right)
$$

where the fit for firm $o$ is given by the minimum value of deviation across all configurations $i$. The fit can also be visualized as seen in Figure 3.

\section{Summary}

An assessment methodology of organizational effectiveness of the architectu$\mathrm{ral} /$ construction firm has been developed and proposed using the configurational theory of organizations and the competing values approach model of organizational effectiveness. The competing values approach analysis of effectiveness criteria along two three dimensions of flexibility, organizational focus, and strategic means and ends by which the organization achieves its goals, is used to 
hypothesize four effective configurations of organizational characteristics. Depending on the particular time in the life cycle of the firm, each effective configuration has different level of criteria that relate to organizational structural flexibility, organizational focus, and strategic means and ends by which the organization achieves its goals. This paper outlines criteria that underlie the most significant variables that are deemed relevant for examining effectiveness in the context of the architectural/construction firm. They include the level of subcontracting, level of integration in services offered, multi-project handling ability, level of strategic planning, level of goal setting, level of workers' participation in decision making, strength of organizational culture, extent of using rules and regulation in the workplace, level of adhering to rules and regulations.

There is need for field work to check on the validity of the inclusion of the outlined effectiveness criteria and also that of the proposed assessment model and its reliability. Future research is needed to apply the theorized model that uses the minimum deviation between the levels of identified criteria in the construction firm with that of the levels across all configurations, to yield an assessment for the level of organizational effectiveness in the firm.

\section{References}

[1] Lewin, Y.A. and Minton. W.J., Determining Organizational Effectiveness: Another Look and Agenda For Research. Management Science. Vol. 32 (5): 514 -537 (1986).

[2] Pennings, J.M. and Goodman, P.S., New Perspective On Organizational Effectiveness. Jossey-Bass, Inc., San Francisco, California, (1977).

[3] Hitt, A.M., The Measuring of Organizational Effectiveness: Multiple Domains and Constituencies. Management In Review, Vol. 28: 28-39 (1988).

[4] Tsui. A.S., A Multiple Constituency Model of Effectiveness: An Empirical Examination of the Human Resource Subunit Model: Administrative Science Quarterly, Vol. 35: 458-483 (1990).

[5] Miller, D. and Friesen, H.P., Organizations: A Quantum View. Prentice-Hall, (1984).

[6] Miles, R.E. and Snow, C.C., Organizational Strategy, Structure and Process. McGraw-Hill New York, (1978).

[7] Mintzberg, H., The Structuring of Organizations. Prentice-Hall, Englewood Cliffs, NJ, (1979).

[8] Meyer, A.D., Tsui, A.S. and Hinings, C.R., Configurational Approach to Organizational Analysis. Academy of Management Journal, Vol. 36(6): 1175-1195 (1993).

[9] Quinn, R.E. and Rohrbaugh, J., A Spatial Model of Effectiveness: Criteria Towards a Competing Values Approach to Organizational Analysis. Journal of Management Science, Vol. 29: 363-377 (1983).

[10] Cameron, K.S., A Study of Organizational Effectiveness and Its Predictors Management Science, Vol. 32: 87-112 (1986).

[11] Maloney, W.F. and Federle, M.O., Practical Models For Organizational Assessment. ASCE Journal of Management in Engineering. Vol. 9(1): 64-81 (1993). 
[12] Ostroff, C. and Schmitt, N., Configurations of Organizational Effectiveness and Efficiency. Academy of Management Journal, Vol. 36 (6): 1345-1361 (1993).

[13] Dotty, D.H., Glick, W.H. and Huber, G.P., Fit, Equafinality, and Organizational Effectiveness: A Test of Two Organizational Theories. Academy of Management Journal, Vol. 36 (6): 1196-1250 (1993).

[14] Eccles, G. R., The quasifirm in the Construction industry. Journal of Economic Behavior and Organization, Vol. 2: 335-357 (1981).

[15] Krippaehne. C.R., McCullouch. G. B. and Vanegas. A.J., Vertical Business Integration Strategies For Construction. ASCE Journal of Management in Engineering, Vol. 8(2): 153166 (1992).

[16] Handa, V. and Adas, A., Predicting The Level Of Organizational Effectiveness : A Methodology For the Construction Firm. Construction Management and Economics, Vol. 14: 341352 (1996).

[17] Handa, V. and Adas, A., Organizational Effectiveness of The Construction Firm: A Framework for Assessment. The Organization and Management of Construction: Shaping Theory and Practice, Ed. by D.A. Langford and A. Retik, 1: 247-2256 (1996).

[18] McKim , R., Adas, A. and Handa, V., Construction Firm Organizational Effectiveness: A Neural Network-Based Prediction Methodology. The Organization and Management of Construction: Shaping Theory and Practice, Ed. by D.A. Langford and A. Retik, Vol. 3: 247-256 (1996).

\section{Notation}

The following symbols are used in this paper:

$D_{i o}=$ the distance between levels of criteria in configuration $i$ and firm $o$

$X_{i}=\mathrm{a} 1 \mathrm{x} j$ vector that represent the level of configuration $i$ on criteria $j$

$X_{o}=$ a $1 \times j$ vector represent the level of firm $o$ on criteria $j$

Fit $_{o}=$ the level of fit for firm $o$. 


\title{
منهج مبني على الشكل التنظيمي لتقييم فاعلية التنظيم بمؤسسات العمارة و التشييد
}

\author{
عدنان بن عباس بن أحمد عدس
}

قسم العمارة ، كلية تصاميم البيئة ، جامعة الملك عبد المبن العزيز

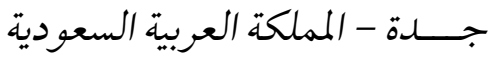

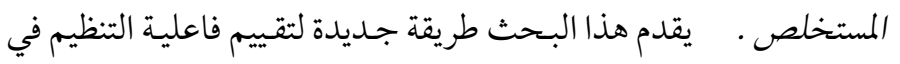

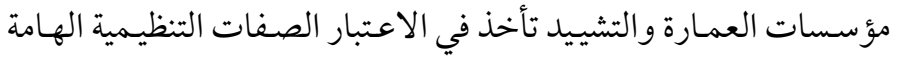

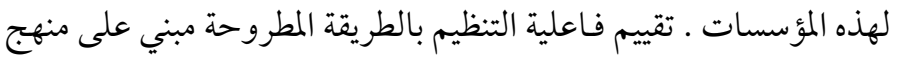

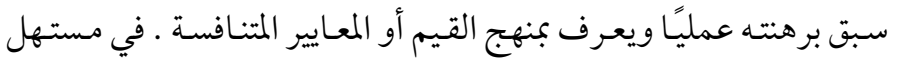

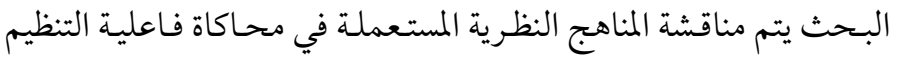

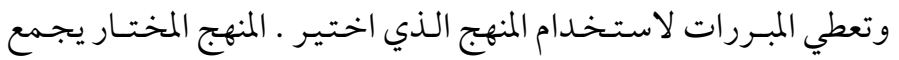

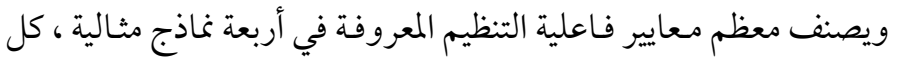

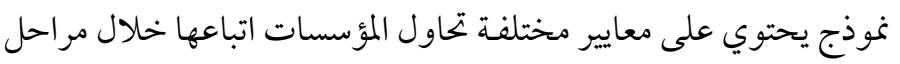

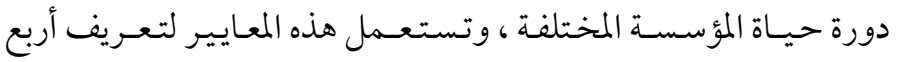

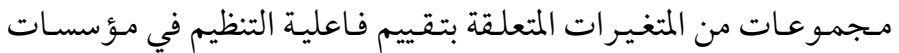

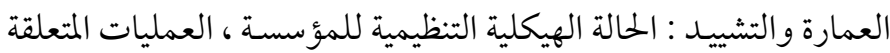

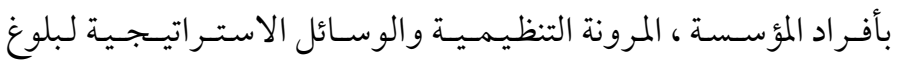

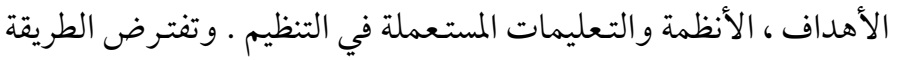

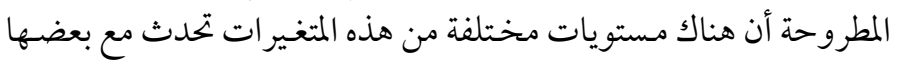

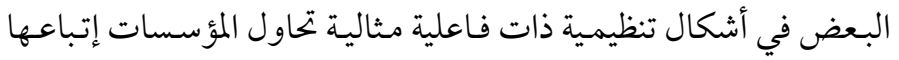

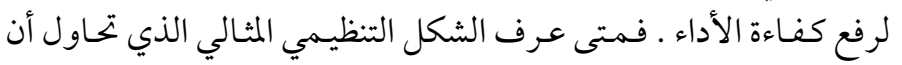

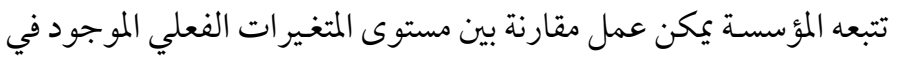

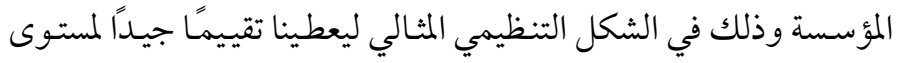

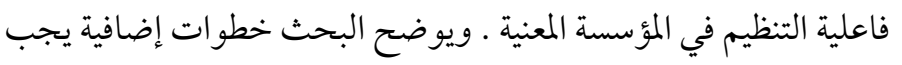

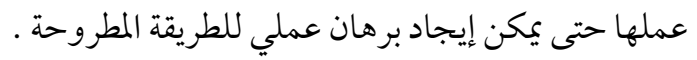

